\title{
Impact of Dental Rehabilitation Under General Anesthesia on Oral Health-Related Quality-of-Life and Dental Anxiety in Turkish Children
}

\author{
Gülsüm Duruk1 (1D, Raziye Kuru¹, Ahmet Selim Özkan² (D)
}

${ }^{1}$ Department of Pediatric Dentistry, Faculty of Dentistry, Inonu University, Malatya, Turkey.

${ }^{2}$ Department of Anesthesiology and Reanimation, Faculty of Medicine, Inonu University, Malatya, Turkey.

Correspondence: Gulsum Duruk, DDS, Ph.D., Department of Pediatric Dentistry, Faculty of Dentistry, Inonu University, Malatya, Turkey. E-mail: durukgulsum@yahoo.com

Academic Editor: Alidianne Fábia Cabral Cavalcanti

Received: 01 June 2020 / Review: 30 July 2020 / Accepted: 08 September 2020

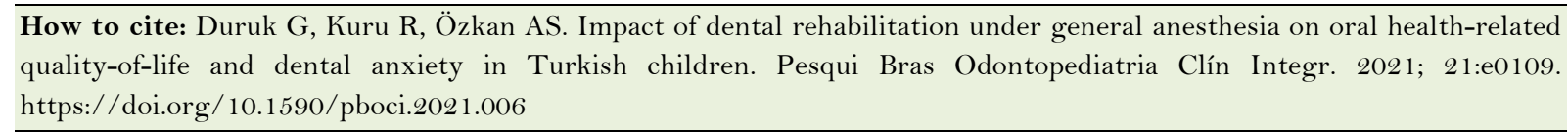

\begin{abstract}
Objective: To analyze children's and parents' oral health-related quality of life (OHRQoL) and children's dental fears before and after the dental rehabilitation under general anesthesia (DRGA). Material and Methods: 104 parents and their 3 to 13-year-old children $(5.90 \pm 2.42)$ who received DRGA were surveyed before and after DRGA. The children were divided into two groups: Group 1 - healthy children $(n=43)$ and Group 2 - children with medical problems $(n=61)$. After recording their socio-demographic information, parents completed a self-administered questionnaire named Early Childhood Oral Health Impact Scale (ECOHIS), which includes two main parts - Child Impact Section (CIS) and Family Impact Section (FIS). On the other hand, the children received a dentist-administered questionnaire named Children's Fear Survey Schedule-Dental Subscale (CFSS-DS) and Frankle Behavior Scale (FBS). For statistical analyses, Wilcoxon Signed-Rank, Mann Whitney-U, Kruskal-Wallis, and Spearman's Correlation tests were used. Results: A statistically significant decrease in all CIS, FIS, ECOHIS and CFSS-DS scores was observed after DRGA $(\mathrm{p}<0.01)$. This decline was greater in healthy children than in children with systemic problems $(\mathrm{p}<0.01)$. Conclusion: Children's and parents' OHRQoL showed better results after DRGA. The decreases in dental anxiety in children were observed after DRGA.
\end{abstract}

Keywords: Oral Health; Child; Quality of Life; Dental Anxiety; General Anesthesia. 


\section{Introduction}

Dental caries is one of the most prevalent chronic diseases among children [1]. Early childhood caries (ECC) is a common health problem that is described as one or more decayed, missing or filled tooth surfaces in any primary teeth in children the birth to seventy-one months of age [2]. Functional, social and psychological problems due to dental caries affect children and their families negatively and reduce their quality of life [1]. Preschool children are reluctant for treatment because of extensive caries, long-lasting treatment sessions and high levels of dental anxiety [3,4]; moreover, behaviour changes are associated with age [5]. In addition, children with special health care needs (CSHCN) have congenital or acquired disabilities that can be physical, developmental, mental or behavioural and need regular medical controls, specialized programs and services $[6,7]$. Thus, dental rehabilitation under general anesthesia (DRGA) may be preferred for non-cooperated, very young aged children and/or CSHCN [5,8]. DRGA is a good treatment option that can be performed in a single session and in a controlled environment in all cases. DRGA also provides pain-free treatment, which decreases dental anxiety [9].

Dental problems in children and adolescents affect daily life and psychological status of patients and their families [10]. Performing all dental treatments under general anesthesia has brought the idea of measuring the change in Oral Health Related Quality of Life (OHRQoL) [5,11-14]. Early Childhood Oral Health Impact Scale (ECOHIS) can be used to assess OHRQoL before and after the rehabilitation procedures [15]. Although the ECOHIS was initially used among the children aged 6-14 [15-18], it was developed for children aged 0-5 years who could not answer the questionnaire [15]. Thus, Family Impact Scale (FIS) was defined. The ECOHIS was constituted in the United States [15], but it has been adapted to use among children in other countries such as French [19], Chinese [20], Swedish [8], Lithuanian [11] children. Thus, the ECOHIS, which was proved by Peker et al. [21] for the validity and reliability in Turkish population, have been used in Turkey.

Dental anxiety among children, which is recognized as a public health problem in many countries, has a multifactorial etiology [4]. The dental subscale of the children's fear survey schedule (CFSS-DS) is one of the fear survey schedules to evaluate dental anxiety in children. CFSS-DS was developed by Cuthbert and Melamed [22] and validated in Turkish language [23]. Besides, the Frankle Behavior Scale (FBS) is one of the commonly used anxiety scales in children [24].

The aim of this study was to evaluate the effects of general anesthesia in dental treatment on the quality of life, dental fear, and dental anxiety in healthy children and children with systemic diseases.

\section{Material and Methods}

Study Design and Sampling

This study is a prospective cross-sectional study. The minimum sample size required to detect a significant difference in ECOHIS scores was calculated to be at least 39 in each group, (78 in total), considering type I error (alfa) of 0.05 , power (1-beta) of 0.8 , an effect size of 0.64 and two-sided alternative hypothesis $\left(\mathrm{H}_{1}\right)$.

One hundred and four children aged 3 to 13 who received DRGA at Department of Pediatric Dentistry, Inönü University, Turkey, between January 2019 and December 2019 were included in the study. 
CSHCN and the children who did not accept the conventional treatment in the clinic because they had dental anxiety or they were too young were included in this study, while the children who were unable to perform the CFSS-DS questionnaire due to their mental retardation, the children who previously received DRGA, and the children whose parents did not complete the questionnaire were excluded in this study.

\section{Data Collection}

The patients applied to the pedodontics clinic one week before the operation. A survey with questions about socio-demographic status, quality of life, and dental anxiety was applied to the children and their parents (63-mother, 41-father) under the supervision of the pediatric dentist, and then they were transferred to the anesthesia pre-operative clinic.

The Turkish version of ECOHIS scale, which had been proven to be valid and reliable by Peker et al. [21], was filled out by the parents under the supervision of a pediatric dentist, and the children responded to the questions in an interview. The ECOHIS based on 13 items were divided into the Child Impact Section (CIS) and the Family Impact Section (FIS). The CIS includes 4 subsections (9 items): symptoms (1 item), function (4 items), psychology ( 2 items), and self-image and social interaction ( 2 items). The FIS includes 2 subsections (4 items): parental distress ( 2 items) and family function ( 2 items). The ECOHIS items were scored according to the answers from o to 5 (O: “never”, 1: "hardly ever”, 2: “occasionally”, 3: "often”, 4: “very often”, 5 : “don't know"). The score ranges for the total ECOHIS, CIS, and FIS are from 0 to 52, from 0 to 36, and from 0 to 16 , respectively. The "don't know" responses were recorded as missing. The recommendations in the original version of the survey were followed for data scoring [15]. When there were two missing responses in the CIS and/or one missing response in the FIS, these values were imputed using the other responses' mean value in the corresponding section. Participants with more missing values were excluded from the analysis [15,21]. A higher ECOHIS data score indicates greater impact and/or more problems for OHRQoL [15].

The Turkish version of CFSS-DS, which had been proven to be valid and reliable by Serim-Yıldız and Erdur-Baker [23], was carried out in children by a pediatric dentist (GD or RK) and the parents and children responded to the questions in an interview. The scale includes 15 items related to various aspects of dental treatment. Each item can be scored on a 5 point scale from 1 (not afraid) to 5 (very afraid). The total score range was $15-75$. The scores within the range 15-31 indicate a low level of anxiety, while the scores within the range 32-38 indicate a medium level of anxiety, and the scores within the range 39 or more indicate a high level of anxiety [22,23].

The FBS was carried out in children by a pediatric dentist (GD or RK). The scale divides the child's behavior into four categories, ranging from "definitely negative" to "definitely positive" (range from 1 to 4 ) [24-26].

A medical examination was performed when the children came to the anesthesia clinic with their parents for pre-operative examination by a physician under the supervision of a specialist anesthetist (ASO), and the parents responded to the questions about medical history in an interview. The patients were categorized according to the American Society of Anesthesiologists (ASA) classification [27].

The 104 children were divided into two groups. Group 1 consisted of 41 healthy (ASA I), noncooperated children, and group 2 was $63 \mathrm{CSHCN}$ (epilepsy, congenital cardiac anomaly, hyperthyroidism, 
hearing impairment, etc.) (ASA II - with mild systemic disease: $\mathrm{n}=29$ (27.9\%) and ASA III - with severe systemic disease: $\mathrm{n}=32(30.8 \%))$.

On the day of the operation, standard anesthesia monitorization was performed peri-operatively to all the patients, such as non-invasive blood pressure, peripheric oxygen saturation, heart rate and electrocardiography. The patients were premedicated in a different room 20 minutes before the induction of anesthesia. If the patient was provided with intravenous cannulation for the induction of anesthesia, premedication was achieved intravenously. If not provided, oral or nasal route was preferred. Nasal route is often routinely preferred in our anesthesia clinic for premedication. Midazolam was administered at a dose of $0.5 \mathrm{mg} / \mathrm{kg}, 0.5 \mathrm{mg} / \mathrm{kg}$, and $0.1 \mathrm{mg} / \mathrm{kg}$ with oral, nasal, and intravenous route, respectively. Twenty minutes after the premedication, the patient was transferred to the operating room. Standard anesthesia induction was preferred and sevoflurane $8 \%$ and $\mathrm{N}_{2} \mathrm{O} 30 \%$ in oxygen with a facemask were used for the anesthesia induction. The child was intubated with a nasoendotracheal tube after administrating muscle relaxant (rocuronium). Tramadol at a dose of $1 \mathrm{mg} / \mathrm{kg}$ or paracetamol at a dose of $15 \mathrm{mg} / \mathrm{kg}$ was administered for post-operative analgesia. For each child, hemodynamic data were noted during anesthesia.

The clinical dental examinations were carried out with the assistance of a mouth mirror and a probe in a dental chair under the light of a reflector during DRGA by an experienced pediatric dentist (GD or RK). Before the oral examination, the examiners were trained and calibrated to perform the DMFT/dmft score examination. The intra-examiner reliabilities were recorded as 0.983 and 0.978 , respectively, and the interexaminer reliability was 0.985 (ICC $>0.90$ is excellent). DMFT/dmft scores were recorded, but any missing teeth not caused by dental caries were not included in DMFT/dmft score. The necessary dental treatments (fillings, pulp amputations, root canal treatments, extractions, scaling and polishing, etc.) and preventive applications (fissure sealant, fluoride varnish) were performed to all the patients.

After the surgery, the patient was extubated and kept in the recovery room under observation. None of the patients had any medical complications during DRGA or in the recovery room. The patients were discharged with the permission of the anesthesiologist. All the parents and the children were given oral hygiene training before the patients were discharged from the hospital.

One month after the operation, the children were called again to the pedodontics clinic with their parents, and the ECOHIS, CFSS-DS, and FBS sections of the questionnaire were repeated. The same parents (63-mother, 41-father), who completed baseline, completed the follow-up surveys. For the children and the illiterate parents, the questionnaire was read aloud and their responses were recorded by the examiner (GD or RK). In addition, these same parents completed the questionnaires under the supervision of the same pediatric dentist. The data were recorded by the same pediatric dentist (GD or RK).

The mean scores before and after ECOHIS for each section were calculated. For each section, the change in scores after the DRGA was calculated by subtracting the mean post-operative scores from the mean pre-operative scores. Negative change scores indicated an improvement in OHRQoL, while positive change scores indicated deterioration [15,21,28].

The effect sizes (ESs) were calculated by dividing the mean of change scores by the standard deviation of the baseline scores [29]. An effect of $<0.2$ indicated a small but clinically meaningful magnitude of change, 0.2-0.7 a moderate change, and $>0.7$ a large change [29]. 
Statistical Analysis

IBM SPSS software (version 21.0 for Windows, IBM Corp., Armonk, NY, USA) was used for data analyses. Kolmogorov-Smirnov $(\mathrm{n}>50)$ and Shapiro-Wilk $(\mathrm{n}<50)$ were applied to the test for a normal distribution. The pre-treatment and post-treatment scores were compared using the Wilcoxon Signed-Rank test. Mann Whitney-U and Kruskal-Wallis tests were used for comparison between the groups. Spearman's Correlation test was used to measure the correlation between the change score of ECOHIS/CFSS-DS and DMFT/dmft. Also, Multiple Linear Regression test was used to determine the effect of socio-demographic characteristics on OHRQoL. The significance level was accepted as $\mathrm{p}<0.05$.

\section{Ethical Clearance}

The study was conducted after obtaining approval from local ethics committee of Medical School (2019/178). The children's parents were well informed on the purpose of the study and they all signed a consent form.

\section{Results}

The findings of socio-demographic status are presented in Table 1.

Table 1. Socio-demographic status.

\begin{tabular}{|c|c|c|}
\hline \multicolumn{2}{|c|}{ Variables } & \multirow[t]{2}{*}{ N (\%) } \\
\hline Age (Mean and SD) & $5.90 \pm 2.42$ & \\
\hline \multicolumn{3}{|l|}{ Sex } \\
\hline Girl & & $41(39.4)$ \\
\hline Boy & & $63(60.6)$ \\
\hline \multicolumn{3}{|l|}{ Medical Condition } \\
\hline Healthy & & $43(41.3)$ \\
\hline $\mathrm{CSHCN}$ & & $61(58.7)$ \\
\hline \multicolumn{3}{|l|}{ Number of Siblings } \\
\hline 1 & & $12(11.5)$ \\
\hline 2 & & $41(39.4)$ \\
\hline 3 & & $30(28.8)$ \\
\hline $4 \geq$ & & $21(20.2)$ \\
\hline \multicolumn{3}{|l|}{ Order of Birth } \\
\hline $1 \mathrm{st}$ & & $50(48.1)$ \\
\hline 2nd or Other & & $54(51.9)$ \\
\hline Paternal Age (Mean and SD) & $39.13 \pm 5.52$ & \\
\hline Maternal Age (Mean and SD) & $34.48 \pm 6.48$ & \\
\hline Age of the Parent Completing & re (Mean and SD) & $36.58 \pm 6.47$ \\
\hline \multicolumn{3}{|l|}{ Paternal Education } \\
\hline Illiterate & & $2(1.9)$ \\
\hline Primary School & & $25(24.0)$ \\
\hline Secondary School & & $7(6.7)$ \\
\hline High School & & $35(33.7)$ \\
\hline University & & $35(33.7)$ \\
\hline \multicolumn{3}{|l|}{ Maternal Education } \\
\hline Illiterate & & $10(9.6)$ \\
\hline Primary School & & $55(52.9)$ \\
\hline Secondary School & & $9(8.7)$ \\
\hline High School & & $\mathrm{o}(0.0)$ \\
\hline University & & $30(28.8)$ \\
\hline
\end{tabular}




\begin{tabular}{|c|c|}
\hline \multicolumn{2}{|l|}{ Education Level of the Parent Completing the Questionnaire } \\
\hline Illiterate & $6(5.8)$ \\
\hline Primary School & $38(36.5)$ \\
\hline Secondary School & $7(6.7)$ \\
\hline High School & $12(11.5)$ \\
\hline University & $41(39.4)$ \\
\hline \multicolumn{2}{|l|}{ Place of Residence } \\
\hline City Center & $91(87.5)$ \\
\hline Suburb & $13(12.5)$ \\
\hline \multicolumn{2}{|l|}{ Patients' Location } \\
\hline In the Same City as the General Anesthesia Center & $57(54.8)$ \\
\hline In a Different City from the General Anesthesia Center & $47(45.2)$ \\
\hline \multicolumn{2}{|l|}{ Income Level } \\
\hline Low $(<2000$ TRY) & $46(44.2)$ \\
\hline Medium (2000-5000 TRY) & $53(51.0)$ \\
\hline High $(>5000$ TRY) & $5(4.8)$ \\
\hline DMFT/dmft score (Mean and SD) & $11.19 \pm 3.65$ \\
\hline
\end{tabular}

Of the patients, 144 were treated under general anesthesia, but 40 of them $(27.8 \%)$ were excluded from the study (72.2\% with follow up rate) because the parents of 25 patients did not fill out the survey for some reason before or after the treatment, and 15 of them missed more than two items in the CIS or more than one item in the FIS.

The rest 104 children (63 boys and 41 girls) whose mean ages were $5.90 \pm 2.42$ (range 3 to 13 years) were included in this study. The survey was filled out by $40.4 \%$ fathers (mean age: $34.48 \pm 6.48$ years) and $59.6 \%$ mothers (mean age: $39.13 \pm 5.52$ years). Of the children, $41.3 \%$ were healthy (ASA I) (mean age: $4.42 \pm$ 1.44), while $58.7 \%$ of them had systemic problems (ASA II and III) (mean age: $6.95 \pm 2.43$ ).

The mean DMFT/dmft score before the treatment was $11.19 \pm 3.65$ (range: 5-20). It was $10.93 \pm$ 3.48 in healthy children and $11.38 \pm 3.78$ in $\mathrm{CSHCN}(\mathrm{p}>0.05)$.

As illustrated in Table 2, the baseline ECOHIS scores were significantly higher than the ECOHIS scores after the treatment. Following the DRGA, in all of the questionnaire sections, there was a significant reduction in the ECOHIS scores $(\mathrm{p}<0.001)$. The reductions in CIS and FIS scores following the treatment were $87.5 \%$ and $91.2 \%$, respectively, and there was an $88.7 \%$ reduction in the total ECOHIS score.

As for the magnitude of change, the total ECOHIS, the CIS, and the FIS demonstrated a large magnitude of change. The ES for each section was large, except for "child self-image" (0.1). The largest ES was for "child symptoms" (2.9), followed by "parental distress" (1.7) (Table 2).

According to the CIS score, no change was observed in the results of 2 children, while the results in one child showed deterioration. Based on the FIS score, no change was observed in 5 parents, while deterioration was detected in 1 parent's score. No change was observed in the results of 3 children according to the total ECOHIS score. In healthy children group, in baseline, CIS, FIS, ECOHIS scores, the "child selfimage" and "parental distress" subsections were higher than in CSHCN group ( $<<0.01)$.

The spearman correlation coefficients between the CIS and FIS scores were in baseline; $r=0.464$, $\mathrm{p}<0.001$ and after treatment; $\mathrm{r}=0.439, \mathrm{p}<0.001$.

The socio-demographic variables determined in the regression model explain $2.9 \%$ of the variances in the change score of ECOHIS (before ECOHIS - after ECOHIS) (Table 3). Although the "education level of the parent completing the questionnaire" variable had the most explanatory power $(\beta=0.234)$, there was no 
statistically significant difference in the change score of ECOHIS in terms of this variable ( $>>0.05)$. The "ASA" variable had the second most explanatory power $(\beta=0.143)$, and there was a statistically significant difference in the change score of ECOHIS between ASA I and ASA II $(p<0.05)$.

Table 2. Mean total and subscale ECOHIS scores at baseline and follow-up and their observed effect mean differences and effect sizes.

\begin{tabular}{|c|c|c|c|c|c|}
\hline Variables & $\begin{array}{c}\text { Pre-Treatment } \\
\text { Mean (SD) } \\
\end{array}$ & $\begin{array}{c}\text { Post-Treatment } \\
\text { Mean }(\mathrm{SD})\end{array}$ & p-value* & $\begin{array}{l}\text { Change in } \\
\text { Score (SD) }\end{array}$ & $\begin{array}{c}\text { Effect } \\
\text { Size }\end{array}$ \\
\hline \multicolumn{6}{|l|}{ Healthy Children $(\mathrm{n}=43)$} \\
\hline CIS & $15.49(6.71)^{\mathrm{a}}$ & $2.00(3.61)$ & $<0.001$ & $-13.49(6.79)$ & +2 \\
\hline Child Symptoms & $3.44(0.96)$ & $0.16(0.43)$ & $<0.001$ & $-3.28(1.08)$ & +3.4 \\
\hline Child Function & $6.67(3.16)$ & $1.16(2.01)$ & $<0.001$ & $-5.51(3.53)$ & +1.7 \\
\hline Child Psychology & $4.44(2.73)$ & $0.12(0.50)$ & $<0.001$ & $-4.33(2.82)$ & +1.6 \\
\hline Child Self-image and Social Interaction & $0.93(2.28)^{\mathrm{a}}$ & $0.56(1.82)$ & 0.320 & $-0.37(2.32)$ & +0.16 \\
\hline FIS & $8.07(3.39)^{\mathrm{a}}$ & $0.49(1.28)$ & $<0.001$ & $-7.58(2.89)$ & +2.2 \\
\hline Parental Distress & $5.23(2.20)^{\mathrm{a}}$ & $0.28(0.91)$ & $<0.001$ & $-4.95(2.10)$ & +2.3 \\
\hline Family Function & $2.84(2.52)$ & $0.21(0.64)$ & $<0.001$ & $-2.63(2.38)$ & +1 \\
\hline ECOHIS & $23.56(8.71)^{\mathrm{a}}$ & $2.49(3.86)$ & $<0.001$ & $-21.07(8.53)$ & +2.4 \\
\hline \multicolumn{6}{|l|}{$\operatorname{CSHCN}(\mathrm{n}=61)$} \\
\hline CIS & $12.77(5.70)^{\mathrm{b}}$ & $1.54(3.16)$ & $<0.001$ & $-11.23(6.30)$ & +2 \\
\hline Child Symptoms & $3.16(1.10)$ & $0.34(0.81)$ & $<0.001$ & $-2.82(1.23)$ & +2.6 \\
\hline Child Function & $5.74(3.21)$ & $0.75(1.54)$ & $<0.001$ & $-4.98(3.51)$ & +1.6 \\
\hline Child Psychology & $3.72(2.37)$ & $0.31(0.89)$ & $<0.001$ & $-3.41(2.49)$ & +1.4 \\
\hline Child Self-image and Social Interaction & $0.15(0.68)^{\mathrm{b}}$ & $0.13(0.81)$ & 0.786 & $-0.02(1.07)$ & +0.03 \\
\hline FIS & $6.16(3.46)^{\mathrm{b}}$ & $0.71(1.82)$ & $<0.001$ & $-5.46(3.22)$ & +1.6 \\
\hline Parental Distress & $3.95(2.49)^{\mathrm{b}}$ & $0.41(1.22)$ & $<0.001$ & $-3.54(2.43)$ & +1.4 \\
\hline Family Function & $2.21(2.15)$ & $0.30(0.78)$ & $<0.001$ & $-1.92(2.01)$ & +0.9 \\
\hline ECOHIS & $18.93(7.72)^{b}$ & $2.25(4.61)$ & $<0.001$ & $-16.69(8.28)$ & +2.2 \\
\hline \multicolumn{6}{|l|}{ Total $(n=104)$} \\
\hline CIS & $13.89(6.25)$ & $1.73(3.34)$ & $<0.001$ & $-12.16(6.57)$ & +2 \\
\hline Child Symptoms & $3.28(1.05)$ & $0.27(0.69)$ & $<0.001$ & $-3.01(1.19)$ & +2.9 \\
\hline Child Function & $6.13(3.21)$ & $0.92(1.75)$ & $<0.001$ & $-5.20(3.51)$ & +1.6 \\
\hline Child Psychology & $4.02(2.55)$ & $0.23(0.75)$ & $<0.001$ & $-3.79(2.65)$ & +1.5 \\
\hline Child Self-image and Social Interaction & $0.47(1.60)$ & $0.31(1.33)$ & 0.359 & $-0.16(1.70)$ & +0.1 \\
\hline FIS & $6.95(3.54)$ & $0.62(1.61)$ & $<0.001$ & $-6.34(3.25)$ & +1.8 \\
\hline Parental Distress & $4.48(2.45)$ & $0.36(1.10)$ & $<0.001$ & $-4.13(2.40)$ & +1.7 \\
\hline Family Function & $2.47(2.32)$ & $0.26(0.72)$ & $<0.001$ & $-2.21(2.19)$ & +1 \\
\hline T-ECOHIS & $20.85(8.42)$ & $2.35(4.30)$ & $<0.001$ & $-18.50(8.62)$ & +2.2 \\
\hline
\end{tabular}

*Wilcoxon; Effect size $(-\infty \ldots+\infty)$; 0.2>: Small; 0.2-0.7: Moderate; 0.7<: Large; ${ }^{a, b}$ Mann Whitney U, p $<0.01$; Different letters indicate statistically significant differences; SD: Standard Deviation.

Table 3. Explanation of the OHRQoL with multiple linear regression analysis.

\begin{tabular}{lccccc}
\hline \multicolumn{1}{c}{ Model } & B & Std. Error & $\boldsymbol{\beta}$ & t & p-value \\
\hline (Constant) & 40.828 & 8.574 & & 4.762 & 0.000 \\
Gender & -1.065 & 1.766 & -0.061 & -0.603 & 0.548 \\
Medical Status (Healthy/ CSHCN) & -7.194 & 4.097 & -0.413 & -1.756 & 0.082 \\
ASA Classification & 1.451 & 2.485 & 0.143 & 0.584 & 0.561 \\
Number of Siblings & 1.196 & 1.317 & 0.131 & 0.908 & 0.366 \\
Order of Birth & -3.230 & 2.229 & -0.188 & -1.449 & 0.151 \\
Parent (Mother or Father) Completing the & -3.779 & 2.599 & -0.216 & -1.454 & 0.149 \\
Questionnaire & & & & & \\
Education Level of the Parent Completing the & 1.381 & 1.632 & 0.234 & 0.846 & 0.400
\end{tabular}

Questionnaire 


\begin{tabular}{|c|c|c|c|c|c|}
\hline Paternal Education & -0.345 & 1.315 & -0.049 & -0.263 & 0.793 \\
\hline Maternal Education & -1.846 & 1.299 & -0.308 & -1.422 & 0.159 \\
\hline Place of Residence & -2.003 & 2.835 & -0.077 & -0.706 & 0.482 \\
\hline $\begin{array}{l}\text { Patients' Location in the Same/Diferent City } \\
\text { as/from the General Anesthesia Center }\end{array}$ & 0.320 & 1.797 & 0.019 & 0.178 & 0.859 \\
\hline Income & -1.253 & 2.047 & -0.085 & -0.612 & 0.542 \\
\hline Model Summary & $\mathrm{R}$ & $\mathrm{R}^{2}$ & Adjusted $\mathrm{R}^{2}$ & \multicolumn{2}{|c|}{ SEE } \\
\hline & $0.377^{\mathrm{a}}$ & 0.142 & 0.029 & \multicolumn{2}{|c|}{8.48998} \\
\hline
\end{tabular}

aDependent Variable: The change in ECOHIS score; *Multiple linear regression analysis.

There was a correlation between the change score of ECOHIS and DMFT/dmft score $(r=0.888$, $\mathrm{p}=0.014)$.

There was a statistically significant decrease in CFSS-DS and FBS in both groups and overall $(\mathrm{p}<0.001)$ (Table 4). According to the CFSS-DS, an increase in anxiety level was observed in 19 children, while 14 children showed no anxiety change. The remaining 81 children exhibited a decrease in had reduced anxiety. Based on the FBS, the anxiety level increased in 4 children, did not change in 48 children and decreased in 52 children after DRGA. The percentage of the decrease in CFSS-DS following the treatment was 22.3\%. This shows that the anxiety decreased after the operation in children who had their dental treatments under GA. However, there was no statistically significant correlation between the DMFT/dmft score and the change score of CFSS-DS $(\mathrm{r}=0.035, \mathrm{p}=0.725)$, while there was a negative correlation between the children's ages and the change scores of CFSS-DS $(\mathrm{r}=-0.293, \mathrm{p}=0.003)$. In terms of CFSS-DS and FBS scores, there were no statistically significant differences between groups and between genders.

Table 4. The mean (SD) CFSS-DS and FBS at baseline and follow-up and their observed effect mean differences and effect sizes.

\begin{tabular}{|c|c|c|c|c|c|c|c|}
\hline \multirow[t]{2}{*}{ Group } & \multicolumn{2}{|c|}{ Pre-Treatment } & \multicolumn{2}{|c|}{ Post-Treatment } & \multirow[t]{2}{*}{ p-value* } & \multirow{2}{*}{$\begin{array}{c}\text { Change in } \\
\text { Score (SD) }\end{array}$} & \multirow{2}{*}{$\begin{array}{c}\text { Effect } \\
\text { Size }\end{array}$} \\
\hline & Mean (SD) & Anxiety Level & Mean (SD) & Anxiety Level & & & \\
\hline \multicolumn{8}{|c|}{ CFSS-DS } \\
\hline Healthy & $38.93(14.03)$ & High & $25.67(15.81)$ & Low & $<0.001$ & $13.26(14.95)$ & +1 \\
\hline $\mathrm{CSHCN}$ & $37.11(16.93)$ & Medium & $32.03(16.47)$ & Medium & $<0.001$ & $5.08(11.06)$ & +0.3 \\
\hline Total & $37.87(15.75)$ & Medium & $29.40(16.42)$ & Low & $<0.001$ & $8.46(13.37)$ & +0.5 \\
\hline \multicolumn{8}{|c|}{ FBS } \\
\hline Healthy & \multicolumn{2}{|c|}{$1.33(0.78)$} & \multicolumn{2}{|c|}{$2.51(1.24)$} & $<0.001$ & $-1.19(1.32)$ & -1.5 \\
\hline $\mathrm{CSHCN}$ & \multicolumn{2}{|c|}{$1.61(1.01)$} & \multicolumn{2}{|c|}{$2.18(1.13)$} & $<0.001$ & $-0.57(0.94)$ & -0.6 \\
\hline Total & \multicolumn{2}{|c|}{$1.49(0.92)$} & \multicolumn{2}{|c|}{$2.32(1.19)$} & $<0.001$ & $-0.83(1.14)$ & -0.9 \\
\hline
\end{tabular}

*Wilcoxon; Anxiety Level = 15-31: Low; 32-38: Medium; 39 or More: High; SD: Standard Deviation.

The features of the anesthesia and the analgesia distributions and the evaluation data were shown in Table 5. General anesthesia was preferred for the dental treatment in all the patients. General anesthesia was previously applied to 48 of the patients (46.2\%). Midazolam was administered to all the patients for premedication and appropriately applied to the patients nasally, orally, and intravenously 79 (76\%), 15 (14.4\%), and $10(9.6 \%)$ of the patients, respectively. Paracetamol and tramadol were also administered for post-operative analgesia in $83(79.8 \%)$ and $21(20.2 \%)$ of the patients, respectively.

There was a significant difference between intraoral and intranasal applications from the premedication routine in terms of the change in CFSS and FBS $(\mathrm{p}<0.05)$. 
Table 5. Features of anesthesia and analgesia distributions and CFSS-DS's and FBS's observed effect mean differences and effect sizes.

\begin{tabular}{|c|c|c|c|c|c|c|c|c|c|c|}
\hline \multirow[b]{2}{*}{ Variables } & \multirow[b]{2}{*}{ Categories } & \multirow[t]{2}{*}{$\mathbf{N}$} & \multirow[t]{2}{*}{$\%$} & \multicolumn{2}{|c|}{ Pre-Treatment } & \multicolumn{2}{|c|}{ Post-Treatment } & \multirow{2}{*}{$\begin{array}{l}\text { Change in } \\
\text { Score (SD) }\end{array}$} & \multirow[t]{2}{*}{ p-value* } & \multirow[t]{2}{*}{ p-value $* * *$} \\
\hline & & & & Mean (SD) & Anxiety Level & Mean (SD) & Anxiety Level & & & \\
\hline \multicolumn{11}{|c|}{ CFSS-DS } \\
\hline Anesthesia Type & General Anesthesia & 104 & 100.0 & $37.87(15.75)$ & Medium & $29.40(16.42)$ & Low & $8.46(13.37)$ & $<0.001$ & - \\
\hline \multirow[t]{2}{*}{ History of General Anesthesia } & Yes & 48 & 46.2 & $35.71(16.38)$ & Medium & $29.13(16.31)$ & Low & $6.58(13.30)$ & $<0.001$ & 0.466 \\
\hline & No & 56 & 53.8 & $39.71(15.10)$ & High & $29.64(16.67)$ & Low & $10.07(13.33)$ & $<0.001$ & \\
\hline Premedication Drug & Midazolam & 104 & 100.0 & $37.87(15.75)$ & Medium & $29.40(16.42)$ & Low & $8.46(13.37)$ & $<0.001$ & - \\
\hline \multirow[t]{3}{*}{ Premedication Route } & Nasal & 79 & 76 & $37.97(15.87)$ & Medium & $28.11(16.2)$ & Low & $9.86(12.80)^{\mathrm{a}}$ & $<0.001$ & 0.012 \\
\hline & Oral & 15 & 14.4 & $32.73(17.19)$ & Medium & $32.93(16.2)$ & Medium & $-0.20(13.10)^{b}$ & 0.814 & \\
\hline & Intravenous & 10 & 9.6 & $44.70(10.01)$ & High & $34.30(17.9)$ & Medium & $10.40(14.54)^{a b}$ & 0.024 & \\
\hline \multirow[t]{2}{*}{ Post-Operative Analgesia } & Paracetamol & 83 & 79.8 & $38.73(15.95)$ & High & $29.22(16.62)$ & Low & $9.51(13.28)$ & $<0.001$ & 0.092 \\
\hline & Tramadol & 21 & 20.2 & $34.43(14.82)$ & Medium & $30.14(16.01)$ & Low & $4.29(13.21)$ & 0.050 & \\
\hline \multicolumn{11}{|c|}{ FBS } \\
\hline Anesthesia Type & General Anesthesia & 104 & 100.0 & $1.49(0.92)$ & & $2.32(1.19)$ & & $-0.83(1.14)$ & $<0.001$ & - \\
\hline \multirow[t]{2}{*}{ History of General Anesthesia } & Yes & 48 & 46.2 & $1.63(1.04)$ & & $2.31(1.22)$ & & $-0.69(1.17)$ & 0.001 & 0.428 \\
\hline & No & 56 & 53.8 & $1.38(0.80)$ & & $2.32(1.16)$ & & $-0.95(1.12)$ & $<0.001$ & \\
\hline Premedication Drug & Midazolam & 104 & 100.0 & $1.49(0.92)$ & & $2.32(1.19)$ & & $-0.83(1.14)$ & $<0.001$ & - \\
\hline \multirow[t]{3}{*}{ Premedication Route } & Nasal & 79 & 76 & $1.49(0.92)$ & & $2.47(1.14)$ & & $-0.98(1.07)^{\mathrm{a}}$ & $<0.001$ & 0.016 \\
\hline & Oral & 15 & 14.4 & $1.73(1.16)$ & & $1.80(1.21)$ & & $-0.07(1.28)^{b}$ & 0.854 & \\
\hline & Intravenous & 10 & 9.6 & $1.10(0.32)$ & & $1.90(1.29)$ & & $-0.80(1.14)^{\mathrm{ab}}$ & 0.066 & \\
\hline \multirow[t]{2}{*}{ Post-Operative Analgesia } & Paracetamol & 83 & 79.8 & $1.49(0.93)$ & & $2.40(1.16)$ & & $-0.90(1.14)$ & $<0.001$ & 0.144 \\
\hline & Tramadol & 21 & 20.2 & $1.48(0.93)$ & & $2.00(1.27)$ & & $-0.52(1.12)$ & 0.062 & \\
\hline
\end{tabular}

*Wilcoxon; ***Mann-Whitney U /Kruskal-Wallis; SD: Standard Deviation; a,bDifferent letters indicate statistically significant differences; Anxiety Level = 15-31: Low; 32-38: Medium; 39 or More: High. 


\section{Discussion}

In this study, the effects of the dental treatments under general anesthesia on OHRQoL and the anxiety in children were evaluated, and both the increase in the OHRQoL of the children and the decrease in their dental anxiety were determined after the treatment.

Although there are studies in the literature evaluating the effect of dental treatment under general anesthesia on OHRQoL [5,11-13,30], a study sample comparing healthy children with CSHCN has not been found. In this study, the ECOHIS scores before the dental treatment were found to be lower in CSHCN compared to the healthy children. The reason for this difference is that "Child self-image and social interaction" and "Parental distress" scores were higher in healthy children than in CSHCN. General health conditions of CSHCN, as well as regular visits to doctors, drug use, hospitalization, etc. may have overshadowed dental problems. CSHCN and their families may not have considered dental problems to be the most basic or primary problem.

In our previous study [31], we compared the oral findings of the children with epilepsy and healthy children and found that the oral findings of the children with epilepsy were much worse. In another previous study [32], we found that CSHCN had poor oral health. In this study, DMFT/dmft scores of CSHCN (11.38 \pm 3.78) were higher than those of healthy children $(10.93 \pm 3.48)$, but this difference was not statistically significant.

The results of the questionnaire show that OHRQoL has improved in most children. In parallel with our study, there are studies in the literature indicating that dental treatments under general anesthesia improve the OHRQoL according to the ECOHIS [5,11-14]. This improved OHRQoL can be explained by several advantages of DRGA, such as safety, efficiency, convenience, high-quality restorative and preventive (e.g., fissure sealing) dental treatments, and one session requirement [14]. It should not be forgotten that untreated dental caries represents a real clinical problem and it is associated with poor quality of life. Based on the CIS score, no change was observed in the results for 2 children, while the results for one child showed deterioration. No change was observed in the results for 3 children according to the total ECOHIS score. Although DRGA has a positive effect on a child's OHRQoL, the extraction of heavily decayed and unrecoverable teeth creates functional limitations on nutrition and speech, which may jeopardize the children's ability for social interaction [13,33]. In this study, there was a small change in the "Child self-image and social interaction" section after treatment (ES:0.1), and only 1-month follow-up findings were evaluated. Some children may not have been able to adjust to the new changes such as dental restoration, tooth extraction, etc. in their mouths yet. In two different studies [11,13], the "Child self-image and social interaction" sections were reported to show the least change after general anesthesia.

The largest ES in our study was found in the "Child symptom" section, which is similar to the study by Farsi et al. [13]. The elimination of toothache was directly reflected in the questionnaire scores. However, it should be noted that parents completed the questionnaire and that they had limited knowledge of their children's pain experience [11,34].

While no change was observed in 5 parents according to the FIS score, deterioration was detected in 1 parent's score. Some families may not have realized this completely even if the child's dental problems had disappeared, and they may even have answered the questions by considering that the problem would remain $[11,34]$.

In this study, the same parents completed the questionnaires under the same pediatric dentist's supervision in both periods. Including the same parents in the questionnaire before and after the treatment was 
important in order to prevent inconsistency in the responses, which may arise when different parents participate.

According to the linear regression model analysis, socio-demographic variables could explain only $2.9 \%$ of the improvement in the OHRQoL. The "education level of the parent completing the questionnaire" variable had the most explanatory power $(\beta=0.234)$, followed by the variable "ASA" $(\beta=0.143)$.

Dental fear in children has been recognized in many countries as a public health problem [35]. DRGA is preferred as an alternative to performing dental treatment because of dental fear in children [36]. As the perioperative period, especially for children, is a stressful event, premedication must be applied first and it is estimated that up to $65 \%$ of children experience intense anxiety in the perioperative period and during induction of anesthesia [37]. Premedication is commonly used to reduce perioperative anxiety [37] and facilitate anesthesia [38]. Benzodiazepines, mainly midazolam, are most commonly used as a premedication for anxiolysis [38]. Some studies have shown a reduction in induced anxiety with the use of midazolam [39,40]. In our study, midazolam was preferred for premedication in all the patients and given to them with different routes.

Although it is suggested that different scales should be used in children under 6 years of age to determine their dental anxiety, there are studies using the CFSS-DS [14,36]. In our study, the CFSS-DS, as well as FBS, which is frequently preferred in young children [26], were used in all age groups. Based on the CFSS-DS, it was determined in our study that 19 children experienced an increase in anxiety, whereas 14 children showed no change in anxiety. The remaining 81 children experienced a decrease in anxiety. According to the results of this study, it can be said that DRGA is a procedure that mostly reduces anxiety. Similarly, Güney et al. [12] stated that there was a decrease in anxiety, whereas Cantekin et al. [14] reported that there was an increase in anxiety and Klaassen et al. [36] reported no change in anxiety. The reason for the decrease in anxiety could be that the child knows that dental treatments are finished, and so they will go to the dental clinic only for a follow-up visit.

In our study, the anxiety at younger ages decreased dramatically ( $r=-0.293, \mathrm{p}=0.003)$. Similarly, the decrease in anxiety for younger children was significant in the study carried out by Güney et al. [12].

There was a significant difference between intraoral and intranasal applications from the premedication routine in terms of the change in CFSS and FBS $(\mathrm{p}<0.05)$. However, the children who took midazolam administered orally showed no change in anxiety after dental treatment because they already had more cooperative behavior than other children.

Some limitations should be noted in the following points: First, the study was performed in a small group of patients, and the results may not be generalized to other races and countries outside Turkey because the population in this study was composed of Turkish people. Second, this study was conducted in a follow-up period of four weeks, which is relatively inadequate. Although it is known that the OHRQoL increased by $88.7 \%$ in the first month after the treatment, it is not known how much it will change in the future. Third, the patients could not be fully standardized in terms of administration routes of midazolam.

On the other hand, having the same parents complete the questionnaires eliminated the differences in the parents' opinions, which can indicate the strength of this study. In addition, conducting the questionnaire by both parents and children under the supervision of a pediatric dentist and conducting it in an interview eliminated the difficulties in understanding the questionnaire's content.

\section{Conclusion}


The ECOHIS showed that it is sensitive to DRGA in children. An increase in OHRQoL and a decrease in dental anxiety were observed in healthy children and in CSHCN undergoing dental treatment under general anesthesia after a 1-month follow-up period. We propose a longer follow-up period to test the sustainability of treatment effects and the likelihood of response shift.

\section{Authors' Contributions}

GD (D) https://orcid.org/0000-0002-6756-6637 Conceptualization, Methodology, Formal Analysis, Investigation and Writing - Original Draft. RK (D) --- Conceptualization, Investigation, Resources and Writing - Review and Editing.

ASO (D) https://orcid.org/0000-0002-4543-8853 Investigation and Writing - Review and Editing.

All authors declare that they contributed to critical review of intellectual content and approval of the final version to be published.

\section{Financial Support}

None.

\section{Conflict of Interest}

The authors declare no conflicts of interest.

\section{Acknowledgements}

The authors wish to thank all participants for their invaluable contribution to this study.

\section{Data Availability}

The data used to support the findings of this study can be made available upon request to the corresponding author.

\section{References}

[1] Muntean A, Mesaros AS, Festila D, Mesaros M. Modern management of dental decay in children and adolescents - a review. Clujul Medical 2015; 88(2):137. https://doi.org/10.15386/cjmed-401

[2] American Academy on Pediatric Dentistry. Policy on Early Childhood Caries (ECC): classifications, consequences, and preventive strategies. Pediatr Dent 2018; 40(6):60-2.

[3] Baghdadi ZD. Effects of dental rehabilitation under general anesthesia on children's oral health-related quality of life using proxy short versions of OHRQoL instruments. ScientificWorldJournal 2014; $2014: 308439$. https://doi.org/10.1155/2014/308439

[4] Doğan MC, Seydaoglu G, Uguz S, Inanc BY. The effect of age, gender and socio-economic factors on perceived dental anxiety determined by a modified scale in children. Oral Health Prev Dent 2006; 4(4):235-41.

[5] Jankauskiene B, Virtanen JI, Kubilius R, Narbutaite J. Oral health-related quality of life after dental general anaesthesia treatment among children: a follow-up study. BMC Oral Health 2014; 14(1):81. https://doi.org/10.1 186/1472-6831-14-81

[6] American Academy of Pediatric Dentistry. Clinical guideline on management of persons with special health care needs. Pediatr Dent 2004; 26(7 Suppl):77-80.

[7] American Academy of Pediatric Dentistry. Guideline on Management of Dental Patients with Special Health Care Needs. Pediatr Dent 2016; 38(5):67-72.

[8] Ridell K, Borgström M, Lager E, Magnusson G, Brogårdh-Roth S, Matsson L. Oral health-related quality-of-life in Swedish children before and after dental treatment under general anesthesia. Acta Odontol Scand 2015; 73(1):1-7. https://doi.org/10.3109/00016357.2014.919661

[9] Ramazani N. Different aspects of general anesthesia in pediatric dentistry: a review. Iran J Pediatr 2016; $26(2):$ e2613. https://doi.org/10.5812/ijp.2613

[10] Souza JGS, Souza SE, Noronha MDS, Ferreira EFE, Martins A. Impact of untreated dental caries on the daily activities of children. J Public Health Dent 2018; 78(3):197-202. https://doi.org/10.1111/jphd.12259

[11] Jankauskiene B, Virtanen JI, Narbutaite J. Follow-up of children's oral health-related quality of life after dental general anaesthesia treatment. Acta Odontol Scand 2017; 75(4):255-61. https://doi.org/10.1080/00016357.2017.1286034

[12] Guney SE, Araz C, Tirali RE, Cehreli SB. Dental anxiety and oral health-related quality of life in children following dental rehabilitation under general anesthesia or intravenous sedation: a prospective cross-sectional study. Niger J Clin Pract 2018; 21(10):1304-10. https://doi.org/10.4103/njcp.njcp_150_18 
[13] Farsi DJ, Farsi NJ, El-Housseiny AA, Damanhouri WH, Farsi NM. Responsiveness of the Arabic version of the ECOHIS to dental rehabilitation under general anaesthesia. Int $J$ Paediatr Dent 2018; 28(1):52-61. https://doi.org/10.1111/ipd.12307

[14] Cantekin K, Yildirim MD, Cantekin I. Assessing change in quality of life and dental anxiety in young children following dental rehabilitation under general anesthesia. Pediatric Dent 2014; 36(1):12E-17E.

[15] Pahel BT, Rozier RG, Slade GD. Parental perceptions of children's oral health: the Early Childhood Oral Health Impact Scale (ECOHIS). Health Qual Life Outcomes 2007; 5(1):6. https://doi.org/10.1 186/1477-7525-5-6

[16] Jokovic A, Locker D, Tompson B, Guyatt G. Questionnaire for measuring oral health-related quality of life in eightto ten-year-old children. Pediatric Dent 2004; 26(6):512-8.

[17] Foster Page L, Thomson W, Jokovic A, Locker D. Validation of the child perceptions questionnaire (CPQ11-14). J Dent Res 2005; 84(7):649-52. https://doi.org/10.1177/154405910508400713

[18] Gherunpong S, Tsakos G, Sheiham A. Developing and evaluating an oral health-related quality of life index for children; the CHILD-OIDP. Community Dent Health 2004; 21(2):161-9.

[19] Li S, Veronneau J, Allison PJ. Validation of a French language version of the early childhood oral health impact scale (ECOHIS). Health Qual Life Outcomes 2008; 6(1):9. https://doi.org/10.1186/1477-7525-6-9

[20] Lee GH, Mcgrath C, Yiu CK, King NM. Translation and validation of a Chinese language version of the Early Childhood Oral Health Impact Scale (ECOHIS). Int J Paediatr Dent 2009; 19(6):399-405. https://doi.org/10.1111/j.1365-263X.2009.01000.x

[21] Peker K, Uysal Ö, Bermek G. Cross-cultural adaptation and preliminary validation of the Turkish version of the Early Childhood Oral Health Impact Scale among 5-6-year-old children. Health Qual Life Outcomes 2011; 9(1):118. https://doi.org/10.1186/1477-7525-9-118

[22] Cuthbert M, Melamed BG. A screening device: children at risk for dental fears and management problems. J Dent Child 1982; 49(6):432-6.

[23] Serim-Yildiz B, Erdur-Baker O. Examining the cultural validity of fear survey schedule for children: the contemporary fears of Turkish children and adolescents. J Genet Psychol 2013; 174(4):345-65. https://doi.org/10.1080/00221325.2012.678420

[24] Klinberg G. Dental anxiety and behaviour management problems in paediatric dentistry--a review of background factors and diagnostics. Eur Arch Paediatr Dent 2008; 9(Suppl 1):11-5. https://doi.org/10.1007/BF03262650

[25] Yıldırım C, Akgün Ö, Polat G, Ok M, Altun C, Başak F. Assessment of dental fear in turkish children with the frankl behavior rating Scale (FS) and the sound-eye-Motor (SEM) scale. Gülhane Tip Derg 2016; 58:272-6. https://doi.org/10.5455/gulhane.180391

[26] Buchanan H, Niven N. Validation of a Facial Image Scale to assess child dental anxiety. Int J Paediatr Dent 2002; 12(1):47-52. https://doi.org/10.1046/j.0960-7439.2001.00322.x

[27] Mayhew D, Mendonca V, Murthy BVS. A review of ASA physical status - historical perspectives and modern developments. Anaesthesia 2019; 74(3):373-9. https://doi.org/10.1111/anae.14569

[28] Sakaryali D, Bani M, Cinar C, Alacam A. Evaluation of the impact of early childhood caries, traumatic dental injury, and malocclusion on oral healthuRelated quality of life for Turkish preschool children and families. Niger J Clin Pract 2019; 22(6):817-23. https://doi.org/10.4103/njcp.njcp_581_18

[29] Cohen J. Statistical Power Analysis for the Behavioural Sciences. 2 ${ }^{\text {nd }}$. ed. Hillsdale: Lawrence Erlbaum Associates; 1988.

[30] Faker K, Tostes MA, Paula VAC. Impact of untreated dental caries on oral health-related quality of life of children with special health care needs. Braz Oral Res 2019; 32:e117. https://doi.org/10.1590/1807-3107BOR-2018.vol32.0117

[31] Duruk G, Aksoy H, Gurbuz T, Laloglu E, Tan H: Oral hygiene in children with epilepsy: effect of interleukin-1 beta and VEGF levels in gingival crevicular fluid. Dent Med Probl 2017; 54(1):67-71. https://doi.org/10.17219/dmp/65450

[32] Duruk G, Kuru R, Gorgen VA. A comprehensive survey of dental rehabilitation under general anaesthesia at a dental hospital in Turkey. J Res Med Dent Sci 2020; 8(3):44-9.

[33] Knapp R, Gilchrist F, Rodd HD, Marshman Z. Change in children's oral health-related quality of life following dental treatment under general anaesthesia for the management of dental caries: a systematic review. Int J Paediatr Dent 2017; 27(4):302-12. https://doi.org/10.1111/ipd.12259

[34] Thomas CW, Primosch RE. Changes in incremental weight and well-being of children with rampant caries following complete dental rehabilitation. Pediatric Dent 2002; 24(2):109-13.

[35] Akbay Oba A, Dülgergil ÇT, Sönmez IŞ. Prevalence of dental anxiety in 7-to 11-year-old children and its relationship to dental caries. Med Princ Pract 2009; 18(6):453-7. https://doi.org/10.1159/000235894

[36] Klaassen MA, Veerkamp JS, Hoogstraten J. Young children's Oral Health-Related Quality of Life and dental fear after treatment under general anaesthesia: a randomized controlled trial. Eur J Oral Sci 2009; 117(3):273-8. https://doi.org/10.1111/j.1600-0722.2009.00627.x 
[37] Pasin L, Febres D, Testa V, Frati E, Borghi G, Landoni G, et al. Dexmedetomidine vs midazolam as preanesthetic medication in children: a meta-analysis of randomized controlled trials. Paediatr Anaesth 2015; 25(5):468-76. https://doi.org/10.1111/pan.12587

[38] Patel T, Kurdi MS. A comparative study between oral melatonin and oral midazolam on preoperative anxiety, cognitive, and psychomotor functions. J Anaesthesiol Clin Pharmacol 2015; 31(1):37-43. https://doi.org/10.4103/0970-9185.150534

[39] Kain ZN, MacLaren JE, Herrmann L, Mayes L, Rosenbaum A, Hata J, et al. Preoperative melatonin and its effects on induction and emergence in children undergoing anesthesia and surgery. Anesthesiology 2009; 111(1):44-9. https://doi.org/10.1097/ALN.obo13e3181a91870

[40] Kain ZN, Caldwell-Andrews AA, Krivutza DM, Weinberg ME, Wang SM, Gaal D. Trends in the practice of parental presence during induction of anesthesia and the use of preoperative sedative premedication in the United States, 1995-2002: results of a follow-up national survey. Anesth Analg 2004; 98(5):1252-9. https://doi.org/10.1213/01.ane.0000111183.38618.d8 